\title{
A rare case of Arthrographis kalrae keratomycosis in a non-contact lens wearer
}

\author{
R.C. Chow, ${ }^{1}$ K.W. Khaw, ${ }^{2}$ T.A. Kamalden, ${ }^{1}$ S.Y. Chong, ${ }^{2}$ Y. Yaacob, ${ }^{2}$ \\ 'University of Malaya Eye Research Center \& the Department of Ophthalmology, \\ Faculty of Medicine, University of Malaya, Malaysia; ${ }^{2}$ Department of \\ Ophthalmology, Seri Manjung Hospital, Perak, Malaysia
}

\begin{abstract}
Purpose: Keratomycoses secondary to Arthrographis kalrae are rarely reported. Previous cases involved patients with contact lens wear. Herein we report a rare case of severe fungal keratitis due to Arthrographis kalrae in a non-contact lens wearer.

Methods: Case report.

Results: A 52-year-old male presented with a left paracentral corneal ulcer with stromal infiltrates following contact with a foreign body while riding a motorbike. Initial treatment with intensive topical antibiotic drops failed. Antifungal therapy with hourly topical amphotericin B $0.15 \%$ and fluconazole $0.2 \%$, as well as oral fluconazole was initiated. His ulcer progressed rapidly despite aggressive treatment. This led to a small, peripheral corneal perforation which was treated with corneal gluing and bandage contact lens. He also developed secondary glaucoma. Microbiological cultures of his corneal scrapings revealed Arthrographis kalrae. A single dose of subconjunctival amphotericin B $0.015 \%$ $0.1 \mathrm{~mL}$ was then given. At two months after presentation, he eventually underwent therapeutic penetrating keratoplasty $(P K)$ for non-resolving keratomycosis. Following PK, he was maintained on topical amphotericin B $0.15 \%$, fluconazole $0.2 \%$ and ciprofloxacin $0.3 \%$ four-hourly, together with fusidic acid ointment $2 \%$ twice daily. The graft failed three months post-transplant, albeit with no evidence of residual or recurrent infection.

Conclusion: Arthrographis kalrae-related keratitis may occur in non-contact lens wearer. Management remains clinically challenging because this organism causes severe, rapidly progressive keratitis.
\end{abstract}

Key words: Arthrographis kalrae, keratomycosis, fungal keratits, penetrating keratoplasty

\section{Introduction}

Keratomycosis can lead to devastating ocular morbidity. According to the World Health Organization, corneal ulceration and ocular trauma may be responsible for 1.5 to 2 million new cases of corneal blindness each year. ${ }^{1}$ In Asia, as many as $17-44 \%$ of corneal ulcers are due to fungal pathogens. ${ }^{2}$ Of these, filamentous fungi like Fusarium and Aspergillus species represent the commonest etiologic agent. ${ }^{3}$ Other filamentous fungi like Arthrographis kalrae, can rarely cause keratomycosis. To date, we found three reported cases. ${ }^{4-6}$ These cases shared a striking similarity whereby all three patients developed the corneal ulcer while on contact lens use.

Correspondence: Rhuen Chiou Chow, MD, Department of Ophthalmology, University of Malaya Medical Centre, Lembah Pantai, 59100 Kuala Lumpur, Malaysia.

E-mail:rhuen_chiou@yahoo.com 
To add to the literature on this rare human pathogen, we share our case of $A$. kalrae keratomycosis that occurred in a non-contact lens wearer.

\section{Case report}

A 52-year-old male with well-controlled diabetes mellitus presented with intense left eye pain, mild blurring of vision, redness, and epiphora four days following contact with a foreign body while riding motorcycle along the countryside. He denied a history of previous ocular trauma and contact lens use. His best-corrected visual acuity was 20/20 OD and 20/40 OS. There was a left paracentral corneal ulcer with stromal infiltrate measuring $4.0 \times 3.8 \mathrm{~mm}$. Associated with this was an intense anterior chamber reaction with $4+$ cells. No corneal thinning or satellite lesions were present. Intraocular pressure (IOP) on presentation was $12 \mathrm{mmHg}$. Corneal scrapings were smeared for Gram stain, Giemsa stain and wet mount microscopy with potassium hydroxide $(\mathrm{KOH})$ as well as inoculated directly onto the surface of blood agar, chocolate agar, MacConkey agar and Sabouraud dextrose agar.

He was initially treated with hourly topical gentamicin $0.9 \%$ and cefuroxime $5 \%$. However, within five days, endothelial plaque and hypopyon formed. Based on these clinical features as well as the fact that the foreign body could contain organic material, antifungals were started. For this, hourly topical amphotericin B $0.15 \%$ and fluconazole $0.2 \%$, alongside systemic oral fluconazole were added. At this stage, results from Gram, Giemsa and $\mathrm{KOH}$ stains as well as microbiological cultures were all negative.

Despite the aggressive antimicrobial therapy, his ulcer further deteriorated over the next three weeks evidenced by enlargement of stromal infiltrates with fluffy, indistinct margins measuring $9.2 \times 10.0 \mathrm{~mm}$, formation of a dense stromal abscess and an anterior chamber fungal ball. This was further complicated by secondary glaucoma with an IOP of $31 \mathrm{mmHg}$, necessitating the use of three topical antiglaucoma agents. At one month, a small, peripheral corneal perforation occurred with a positive Siedel test. Cyanoacrylate glue corneal patch was successfully performed and a bandage contact lens was placed. Intraoperative corneal swab yielded no growth.

Meanwhile, microbiological cultures of his initial corneal scraping revealed Arthrographis kalrae (Fig. 1). Subsequently, a single dose of subconjuntival amphotericin B $0.015 \% 0.1 \mathrm{ml}$ was given on top of his topical and oral medications. Due to the protracted clinical course, our patient eventually underwent a therapeutic penetrating keratoplasty (PK) two months after presentation. Postoperatively he developed transient hyphema which resolved spontaneously. Following PK, he was maintained on topical amphotericin B $0.15 \%$, fluconazole $0.2 \%$ and ciprofloxacin $0.3 \%$ four-hourly, as well as fusidic acid ointment $2 \%$ twice daily. Suture infiltrates developed a week after surgery which resolved with intensified topical ciprofloxacin $0.3 \%$. His IOP post PK was maintained at $12-18 \mathrm{mmHg}$ on four antiglaucoma eye drops. However, the graft failed three months post-transplant (Fig. 2). Currently this patient is being maintained on topical chloramphenicol $0.5 \%$ and dexamethasone $0.1 \%$ six-hourly, fucidic acid $2 \%$ ointment twice daily as well as 
three anti-glaucoma agents. He has been followed-up for eighteen months posttransplant with no evidence of residual or recurrent disease.
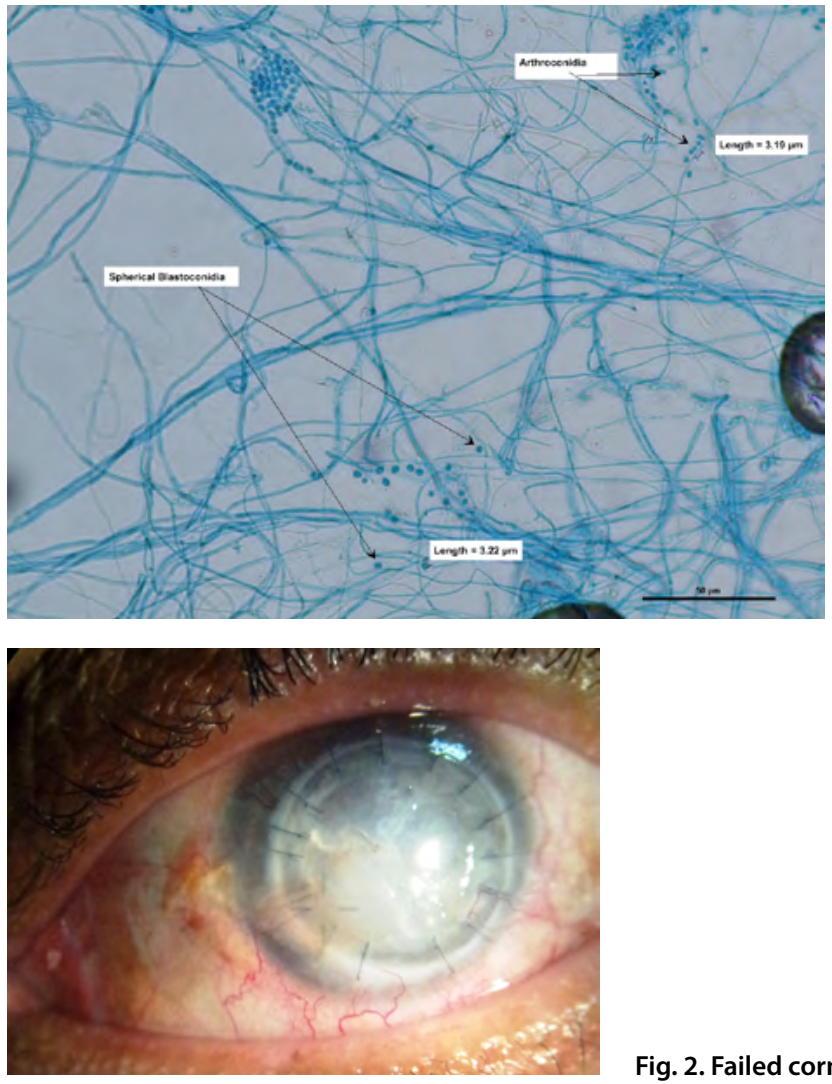

Fig. 1. Arthrographis kalrae isolates from corneal scrapings stained with lactophenol cotton blue showing typical morphological features. One-celled, smooth-walled, arthroconidia are seen.

\section{Discussion}

Arthrographis kalrae, although a rare opportunistic pathogen, has been isolated in a myriad of systemic diseases including pulmonary infection, endocarditis, knee joint infection, sinusitis, meningitis, cerebral vasculitis, panophthalmitis as well as keratomycosis. ${ }^{4-12}$ Since it was first described in 1976 by Cochet ex Sigler and Carmichael, there have been less than 15 clinical case reports of $A$. kalrae-related human infections, including three keratomycoses.

In 1997, Perlman and Binns reported the first case of $A$. kalrae keratitis in a contact lens wearer who was exposed to soil and presented with intense photophobia and progressive infiltrate mimicking Acanthamoeba. ${ }^{4}$ In 2004, Biser et al. highlighted another contact lens-related corneal ulcer that was empirically treated as Acanthamoeba keratitis before fungal culture results grew A. kalrae. ${ }^{5}$ This patient received topical amphotericin $0.4 \%$ and miconazole $10 \%$ and oral itraconazole 200 $\mathrm{mg}$ twice daily. Recently, Thomas et al. reported the third case involving a healthy immune-competent soft contact lens user with rapidly progressing ulcer. Their 
patient underwent three PK and was treated with topical and prolonged systemic voriconazole. $^{6}$

Our patient shares similarities with the above cases in that he had intense eye pain, rapid progressive corneal infiltrate with eventual perforation and the need for PK to control the disease. However, unlike these cases, our patient had never worn contact lenses. Contact lens use has been associated with corneal ulcer via various mechanisms including direct and indirect corneal trauma. ${ }^{13}$ Instead, risk factors for developing fungal keratitis in our patient included diabetes mellitus and possible exposure to organic material as he was traveling along the countryside.

A. kalrae is a slow-growing filamentous fungus found in soil and compost. Due to the rarity of cases, A. kalrae is often misdiagnosed as Candida albicans because of the initial cream-colored, yeast-like appearance. ${ }^{9}$ However, on light microscopy, the characteristic of this species is the presence of one-celled, hyaline, smooth-walled, and cylindrical arthroconidia directly formed by fragmentation of undifferentiated hyphae or for the fresh cultures by disjunction and segmentation of hyaline fertile branches borne at the apex of the conidiophores. ${ }^{11}$ Diagnosis can be challenging and may only be clinched after repeated testing. As such, when performing therapeutic penetrating keratoplasties, the host button should be divided into two parts: the first subjected to microbial analysis; and the other sent for histopathological evaluation. This helps in conforming the diagnosis. Additional testing like polymerase chain reaction may prove helpful. ${ }^{6}$

In terms of therapeutic options, previous sensitivity testing has shown susceptibility of this fungus to amphoterecin, itraconazole and voriconazole. Voriconazole, a noble broad spectrum triazole agent with good ocular penetration, has been effective in treating both common, as well as very rare fungal infections., 5 However, further in-vitro anti-fungal sensitivity testing of this uncommon organism is required for future reference.

In conclusion, this case illustrates that $A$. kalrae keratitis may occur in non-contact lens users. Clinicians should be aware of this rare pathogen when dealing with an intensely painful and rapidly progressive fungal ulcer.

\section{Acknowledgement}

Special thanks to Mr. Alex Francis, Senior Microbiologist, Raja Permaisuri Bainun Hospital, Ipoh, Malaysia, for kindly providing pictures of the microbiology slides. This paper and its contents were written with informed consent of our patient. There is no financial interest to disclose.

\section{References}

1. Whitcher JP, Srinivasan M, Upadhyay MP: Corneal blindness: a global perspective. Bull WHO 2001;79:214-221.

2. Srinivasan M, Fungal keratitis. Curr Opin Ophthalmol 2004;15:321-327.

3. Bharathi MJ, Ramakrishnan R, Vasu S, Meenakshi R, Palaniappan R. Epidemiological characteristics and laboratory diagnosis of fungal keratitis. A three-year study. Indian J Ophthalmol 2003;51:315-321. 
4. Perlman EM, Binns L, Intense photophobia caused by Arthrographis Kalrae in a contact lens-wearing patient. Am J Ophthalmol 1997;123:547-549.

5. Bieser SA, Perry HD, Donnenfeld ED, et al. Arthrographis keratitis mimicking Acanthomoeba keratitis. Cornea 2004;23:314-317.

6. Thomas BC, Zimmermann S, Völcker HE, et al. Severe Arthrographis Kalrae keratomycosis in an immunocompetent patient. Cornea 2011;30:364-366.

7. Vos CG, Murk JL, Hartemink KJ, Daniels JMA, Paul MA, Debets-Ossenkopp YJ. A rare pulmonary infection caused by Arthrographis kalrae. J Med Mircobiol 2012;61(4):593-595.

8. de Diego Candela J, Forteza A, Garcia D, Prieto G, Bellot R, Villar S, Cortina JM. Endocarditis caused by Arthrographis kalrae. Ann Thorac Surg 2010;90(1):e4-5.

9. Boan P, Arthur I, Golledge C, Ellis D. Refractory Arthrographis kalrae native knee joint infection. Med Mycol Case Rep 2012;1:112-114.

10. Chin-Hong PV, Sutton DA, Roemer M, Jacobson MA, Aberg JA. Invasive fungal sinusitis and meningitis due to Arthrographis kalrae in a patient with AIDS. J Clin Microbiol 2001;39(2):804-807.

11. Pichon N, Ajzenberg D, Desnos-Ollivier M, Clavel M, Gantier JC, Labrousse F. Fatal-stroke syndrome revealing fungal cerebral vasculitis due to Arthrographis kalrae in an immunocompetent patient. J Clin Microbiol 2008;46(9):3152-3155.

12. Xi L, Fukushima K, Lu C, Takizawa K, Laio R, Nishimura K. First case of Arthrographis kalrae ethmoid sinusitis and ophthalmitis in the People's Republic of China. J Clin Microbiol 2004;42(10):4828-4831.

13. Ibrahim YW, Boase DL, Cree IA. How could contact lens wearer be at risk of Acanthamoeba? A review. J Optom 2012;2:60-66. 